\title{
Prediction Strategies for Smooth Set Point Modulation to Improve Sensitive DER Response
}

\author{
Mazheruddin H. Syed*, Erfen Guillo-Sansano*, Ali Mehrizi-Sani ${ }^{+}$, Graeme M. Burt* \\ ${ }^{*}$ Institute for Energy and Environment, University of Strathclyde, Glasgow, UK \\ ${ }^{+}$Bradley Department of Electrical and Computer Engineering, Virginia Tech, Blacksburg, VA, US
}

\begin{abstract}
Power systems have been historically expected to operate in a plug-and-play fashion. While in the conventional power system, because of its ample inertia and large generators, frequent small changes in the system may not pose any significant challenge. Moving forward, as (i) the system gets smaller (microgrids) or (ii) the resources become more sensitive to changes (power electronics-based inverters), the need to ensure that the system dynamics are regulated increases to avoid apparatus malfunction, dynamic response challenges, and protection issues. Set point automatic adjustment with correction enabled (SPAACE) is an add-on strategy to improve the performance of an existing controller when the controller itself is black boxed-a common scenario with inverters associated with utility-installed renewable systems. This paper implements a smooth variant of SPAACE in an experimental test bed to evaluate the performance of a proposed linear prediction strategy in several scenarios. This new strategy allows for simpler and faster implementation of SPAACE in practical systems.
\end{abstract}

Index Terms-Control, distributed generation, dynamic response, experimental evaluation, inverter, microgrid, power system, test bed.

\section{INTRODUCTION}

The interconnected electric power system comprises an expansive network of loads, generation sources, and transmission and distribution assets. Historically, the power system with its ample inertia and large generation units is mostly treated as an infinite source that is expected to accommodate small changes in generation and load profiles. However, a number of changes move the power system operation away from the existing dominantly plug-and-play-based paradigm [1]-[3]:

- Smaller power systems. While small amounts of new generation (e.g., a new rooftop PV installation) compare negligible with respect to the total generation available in the terrestrial power system, they may not be considered a small change in other forms of power system such as microgrids. Consequently, the performance of their controllers deteriorates.

- More sensitive resources. Most renewables (PV and wind) are interconnected to the power system via power electronics-based inverters. These inverters are more sensitive to overcurrents and overvoltages than are the traditional synchronous generators. This makes the controller performance deterioration discussed before even more pronounced in such apparatus.

This shift in the operation paradigm of the power system and the associated controllers necessitates careful design of system dynamics and response trajectories to avoid damage
TABLE I. COMPARISON OF EXISTING METHODS TO IMPROVE DYNAMIC RESPONSE OF A SYSTEM

\begin{tabular}{llcc}
\hline Approach & References & No Model & Noninternal \\
\hline PI gain scaling & {$[4],[5]$} & $\sqrt{ }$ & $\mathrm{X}$ \\
Set point ramping & & $\sqrt{ }$ & $\sqrt{ }$ \\
Model predictive control & {$[6]-[8]$} & $\mathrm{X}$ & $\mathrm{X}$ \\
Adding a D-term to PI & {$[9]-[11]$} & $\mathrm{X}$ & $\mathrm{X}$ \\
Extremum seeking & {$[12]-[16]$} & $\sqrt{ }$ & $\mathrm{X}$ \\
Posicast & {$[17]-[22]$} & $\mathrm{X}$ & $\sqrt{ }$ \\
\hline
\end{tabular}

to the apparatus and their components, stability issues, and protection challenges. Ensuring that the controllers that were originally designed for the system continue to operate at their peak dynamic performance can help alleviate these identified issues; however, controller redesign requires availability of system models, system models are difficult to obtain; even when models are available, they risk becoming obsolete with frequent changes in the system. Moreover, controller retuing requires both (i) expert knowledge (to design better parameters) and (ii) access to the controller gains (to update the parameters). However, these requirements are not always available in renewables installations.

Methods that do not require a detailed model of the system and are relatively robust to the system changes can be better suited to this identified control and trajectory design challenge. Table I compares several existing methods based on whether (i) they need the system model, and (ii) they need access to the internal parameters of the existing controller. As can be seen, these methods require one or both. Moreover, once designed, the controller parameters are again appropriate only for a specific operating region; if the parameters of the host system change significantly, the devised controller parameters become irrelevant again, and the whole process needs to be reiterated. Therefore, a scalable approach to autonomously enhance the performance of existing controllers is desired.

Set point automatic adjustment with correction enabled (SPAACE) [23]-[28] is one such strategy that can be used as an add-on controller to improve the performance of existing implemented controllers when they are black boxed. SPAACE achieves this by monitoring the response of an apparatus and modulating its set point to ensure close tracking of the set point. Modulation of the set point is performed by scaling it down (or up) by a preset value. Consequently, SPAACE issues a series of step changes in the system set point. While this approach has proven effective in several power system applications, a smoother and more graceful set point change, 

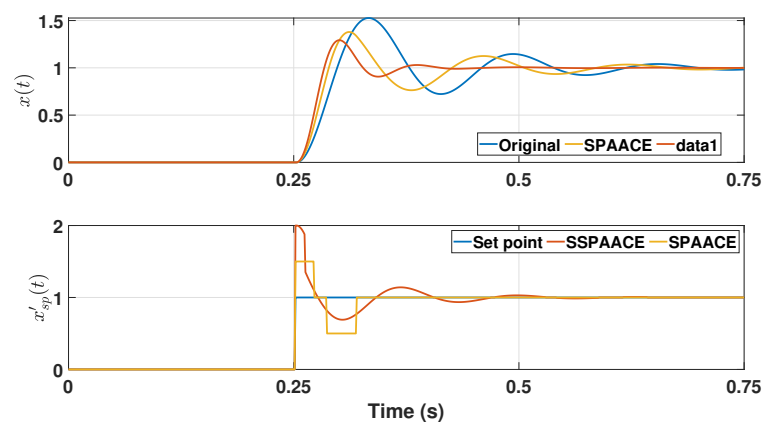

Fig. 1. Example response of a system to a set point change (i) without any add-on feature, (ii) with SPAACE, and (iii) with SSPAACE.

called SSPAACE as originally proposed in [28], may further improve the system response.

This paper builds on this prior work and further improves it via the following contributions:

- Implementation and performance evaluation of a new prediction strategy based on linear formulation to improve the performance of SSPAACE.

- Evaluation of the performance of SSPAACE in an experimental test bed with respect to two different prediction strategies in several scenarios.

The presented case studies demonstrate the superior performance of the proposed linear prediction strategy in terms of overshoot and settling time.

\section{Fundamentals of Smooth SPAACE}

Fig. 1 compares the response of an example system to a step set point change (i) without any add-on feature, (ii) with SPAACE, and (iii) with SSPAACE. The original response has a larger overshoot and longer settling time than either SPAACEand SSPAACE-assisted responses. However, unlike SPAACE, the set point changes imposed by SSPAACE are smooth (hence the name smooth SPAACE), which is more desired when dealing with (i) mechanical systems, or (ii) systems that have poorly tuned controllers. SSPAACE modulates the original set point $x_{\mathrm{sp}}(t)$ of a system by monitoring the tracking error. SSPAACE uses the predicted error, rather than the error itself, to increase the speed of the system response [28]. The change in the set point is determined based on the following control law:

$$
x_{\mathrm{sp}}^{\prime}(t)= \begin{cases}x_{\mathrm{sp}}, & \text { acceptable tracking error } \\ x_{\mathrm{sp}}+m \times e_{\mathrm{pred}}(t), & \text { otherwise }\end{cases}
$$

where $m$ is a scaling factor to penalize tracking error, and $e_{\text {pred }}$ is the predicted tracking error that can be calculated using a predictor by applying the tracking error $e(t)$ defined as

$$
e(t)=x_{\mathrm{sp}}-x(t) .
$$

In this paper, we compare the performance of two predictors: linear and lead compensator. A linear predictor, as proposed in this paper, is based on linear extrapolation of the tracking error. Different linear predictors differ in the number of history terms, the prediction horizon, and the fitting method (normally least-square error (LSE)) [24]. In this work, we use equal history and prediction horizons. With one past data point, the LSE formulation simplifies to an averaging function:

$$
\hat{e}\left(t_{0}+T_{\text {pred }}\right)=2 e\left(t_{0}\right)-e\left(t_{0}-T_{\text {pred }}\right) .
$$

The salient features of the proposed linear predictor are its low (i) computational burden, and (ii) memory requirement.

A lead compensator-based predictor is proposed in [28], where the predicted error in the $s$-domain is calculated as

$$
\hat{e}_{\text {pred }}(s)=\frac{s T+1}{\alpha s T+1)} e(s) .
$$

This lead compensator achieves the prediction function by providing a phase lead in the frequency range $\left[\frac{1}{T}, \frac{1}{\alpha T}\right] . T$ and $\alpha$ are design parameters that determine where the maximum phase lead occurs. As discussed earlier, in our applications, system parameters are not necessarily known; therefore, $T$ and $\alpha$ can be chosen based on past experience. However, due to the adaptive nature of SSPAACE, its performance is not highly dependent on the particular values of $T$ and $\alpha$. However, if system parameters are indeed available, $T$ can be chosen to cancel the dominant pole of the system, hence reducing the overall system order and simplifying its dynamics. Simultaneously, $\alpha$ can be selected to provide a reasonable trade-off between the response speed and noise sensitivity.

In (1), acceptable tracking error is application-specific and normally can be defined as

$$
e_{\min } \leq e_{\text {pred }}(t) \leq e_{\max },
$$

where $e_{\min }$ and $e_{\max }$, normally the same numbers with opposite signs, define the permissible violation range before SSPAACE activates.

\section{STUdY SySTEM}

The performance evaluation of SSPAACE with lead compensator prediction and proposed linear prediction strategies is undertaken at the University of Strathclyde's Dynamic Power Systems Laboratory (DPSL), a $115 \mathrm{kVA}$, low voltage $(400 \mathrm{~V})$ three-phase experimental facility. A simplified oneline diagram of the power network at DPSL is shown in Fig. 2a. A combination of conventional and non-synchronous generation as well as static and dynamic loads allow for representation of land-marine-aero electrical power systems at scale complemented by a NovaCor digital real-time simulator (DRTS) from RTDS Technologies for controller and power hardware-in-the-loop experiments.

\section{A. Test Configuration}

Fig. 2b shows the test configuration utilized for performance evaluation studies in this paper. A simple network, comprising a synchronous generator (SG) and an on load tap changer (OLTC) simulated within RTDS, provides the reference voltage for reproduction by the $90 \mathrm{kVA}$ back-to-back (B2B) power interface. The $15 \mathrm{kVA}$ B2B converter is chosen 


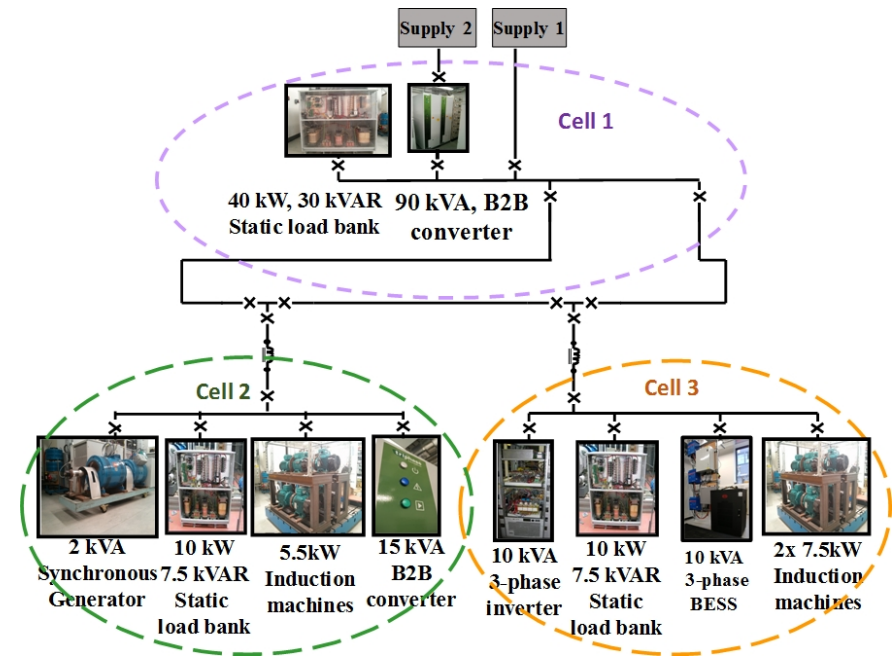

(a) Simplified one line diagram of DPSL.

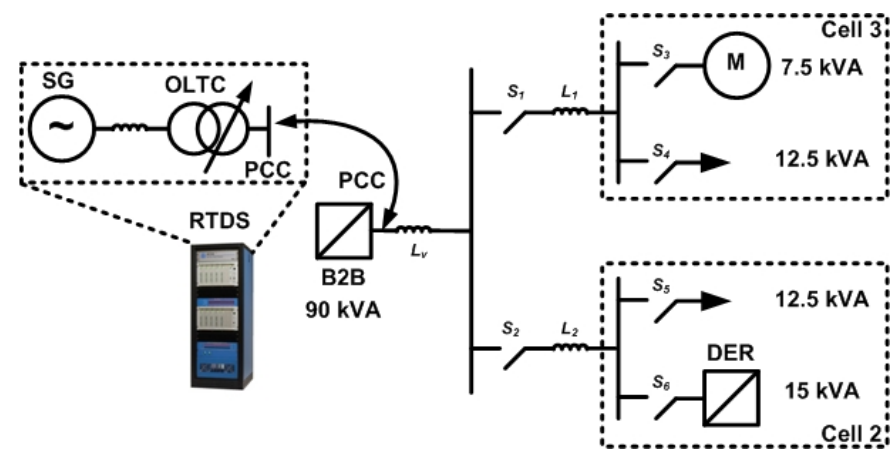

(b) Test configuration.

Fig. 2. Experimental rig and test configuration.

as the distributed energy resource (DER), emulating a power electronics-interfaced energy storage system. A $7.5 \mathrm{kVA}$ induction machine and two $12 \mathrm{kVA}$ static load banks allow testing load switching transients.

\section{B. Implementation of SSPAACE in the Test Bed}

SSPAACE can be incorporated to any DER unit for improvement of its dynamic response, operating either connected to a grid or as a grid forming unit. A grid-connected operation is considered for this paper where the DER operates as a current-controlled voltage source. The incorporation of SSPAACE within the DER controller is shown in Fig. 3, where $V_{a b c}$ are the three-phase instantaneous voltages, $P_{s p}$ and $Q_{s p}$ are the original real and reactive power set points of the DER, $P_{\text {meas }}$ and $Q_{\text {meas }}$ are the measured real and reactive power of the DER, $P_{s p}^{\prime}$ and $Q_{s p}^{\prime}$ are the SSPAACE modified real and reactive power set points of the DER, and $V_{d q}$ and $I_{d q}$ are the direct and quadrature voltages and currents. The parameters for SSPAACE are presented in Table II.

\section{Hardware Dynamic Performance Evaluation}

In this section, the performance of the proposed linear prediction strategy is analyzed with lead compensator prediction strategy and the inherent response of the DER without

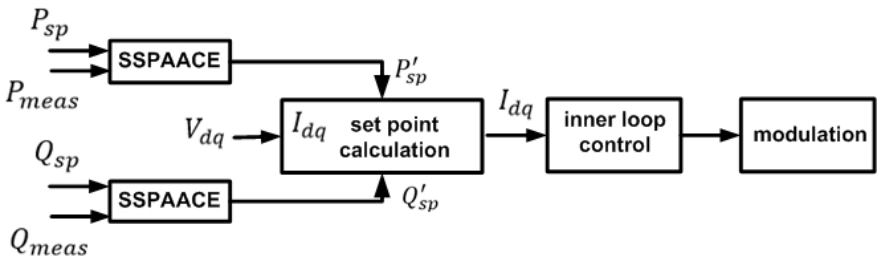

Fig. 3. SSPAACE incorporation within DER controller.

TABLE II. SSPAACE PARAMETERS

\begin{tabular}{cl}
\hline Linear Predictor & Lead Compensator Predictor \\
\hline$m=3.5, T_{\text {pred }}=10$ & $m=3.5, T=0.02 . \alpha=0.05$ \\
\hline$e_{\max }=100 \mathrm{~W}, e_{\min }=-100 \mathrm{~W}$ \\
\hline
\end{tabular}

SSPAACE used as references for comparison. To aid with the assessment, the following key indicators are defined:

- Settling Time: For a step change in the reference set point $x_{s p}$, the settling time $T_{\text {settling }}$ is defined as the time elapsed from the application of the step to the time when $x(t)$ stays within the defined error band as

$$
x_{s p}-\left|e_{\min }\right|<x(t)<x_{s p}+e_{\max } .
$$

- Overshoot: Defining the maximum excursion of $x(t)$ after a step change in reference set point as $x_{\max }$, the overshoot is calculated as

$$
x_{\mathrm{os}}=\left|\frac{x_{\max }-x_{s p}}{x_{s p}}\right|
$$

- Cumulative Tracking Error: Cumulative tracking error is defined as the sum of the the tracking error at every time step $T_{s}$ from the application of the step to the time when the measured output signal has settled at $T_{\text {settling. }}$.

$$
S_{e}=\sum_{k=0}^{N}\left(x_{s p}[k]-x[k]\right) T_{s},
$$

where $N=T_{\text {settling }} / T_{s}$. A smaller $S_{e}$ corresponds to better set point tracking capability. For comparison purposes, we normalize the value of $S_{e}$ to one, corresponding to the non modified response.

For brevity, the performance of the DER is evaluated with respect to only real power. The performance is assessed for four perturbations presented in the following subsections.

\section{A. Case A: Set Point Change in Real Power}

The response of the DER subject to a real power set point change from $2000 \mathrm{~W}$ to $6000 \mathrm{~W}$ is shown in Fig. 4 and the performance indicators presented in Table III. The linear prediction strategy presents a $15.98 \%$ improvement in overshoot over inherent response of the DER and $0.81 \%$ improvement over the lead prediction strategy. Similarly an improvement of $75.4 \%$ and $70.20 \%$ in settling time, respectively over inherent and lead prediction, is observed. 
TABLE III. PERFORMANCE INDICATORS FOR THE FOUR CASES UNDER CONSIDERATION

\begin{tabular}{|c|c|c|c|c|c|c|c|c|c|c|c|c|}
\hline & \multicolumn{2}{|c|}{ Case A } & \multicolumn{2}{|c|}{ Case B } & \multicolumn{4}{|c|}{ Case $\mathrm{C}$} & \multicolumn{4}{|c|}{ Case D } \\
\hline & \multirow[b]{2}{*}{$x_{o s}$} & \multirow[b]{2}{*}{$T_{\text {settling }}$} & \multirow[b]{2}{*}{$x_{o s}$} & \multirow[b]{2}{*}{$T_{\text {settling }}$} & \multicolumn{2}{|c|}{ Step Up } & \multicolumn{2}{|c|}{ Step Down } & \multicolumn{2}{|c|}{ Step Down } & \multicolumn{2}{|c|}{ Step Up } \\
\hline & & & & & $x_{o s}$ & $T_{\text {settling }}$ & $x_{o s}$ & $T_{\text {settling }}$ & $x_{o s}$ & $T_{\text {settling }}$ & $x_{o s}$ & $T_{\text {settling }}$ \\
\hline & $(\%)$ & (s) & $(\%)$ & (s) & $(\%)$ & (s) & $(\%)$ & (s) & $(\%)$ & (s) & $(\%)$ & (s) \\
\hline & 21.58 & 0.32 & 23.97 & 0. & 19. & - & 15. & 0.367 & 8 & 0.1995 & 8.15 & 0.145 \\
\hline Line & 5.6 & 0.07 & 9. & 0.08 & $\sim 1$ & 0.0779 & 4. & 0. & 3. & 0.194 & 2.91 & 0.048 \\
\hline Lead & 6.41 & 0.2668 & 8.82 & 0.1235 & $\sim 1$ & 0.0789 & 6.58 & 0.301 & 3.35 & 0.0541 & 3.33 & 0.055 \\
\hline
\end{tabular}
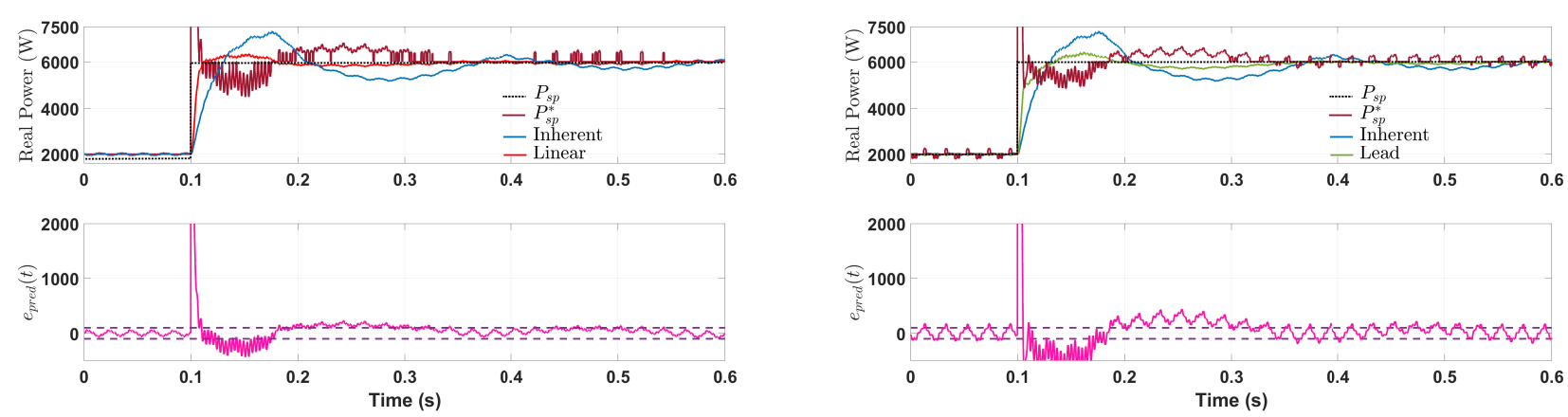

(a) DER response subject to step change in real power (Linear).

(b) DER response subject to step change in real power (Lead).
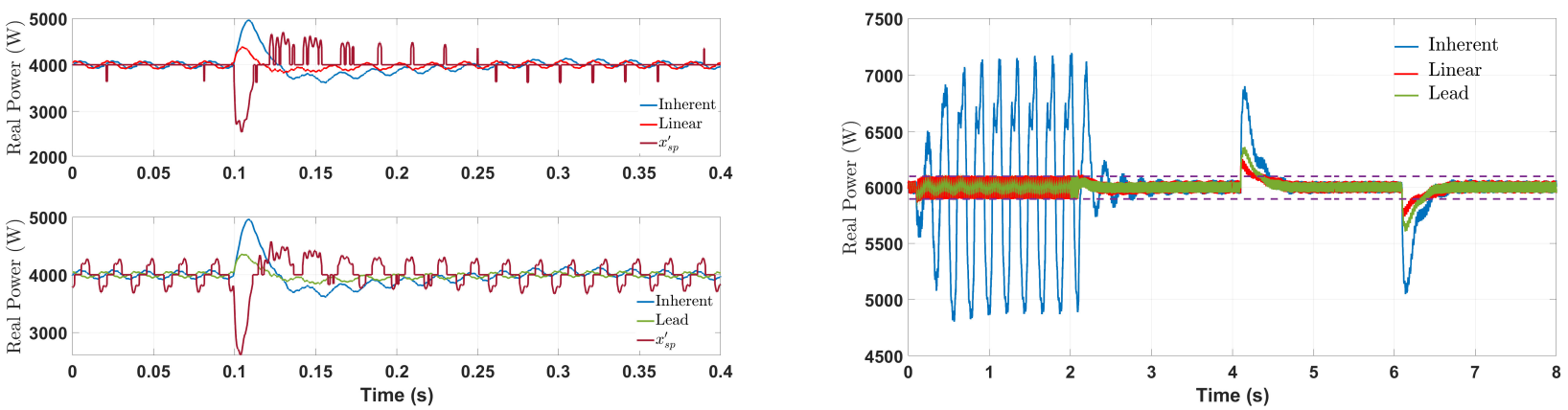

(d) DER response subject to voltage change.

(c) DER response subject to switching transient

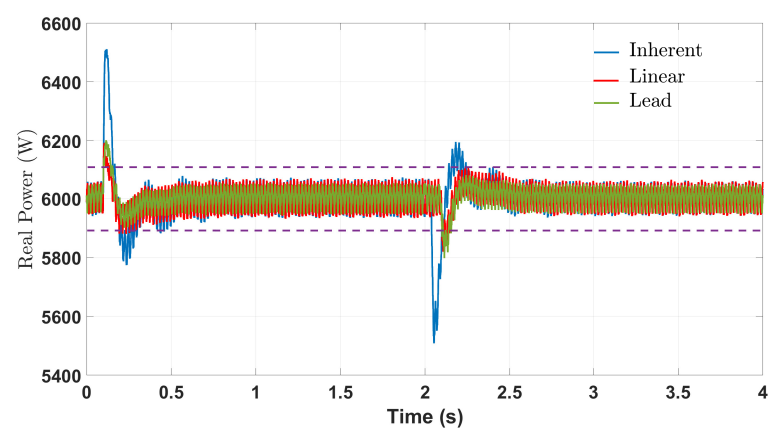

(e) DER response subject to impedance change.

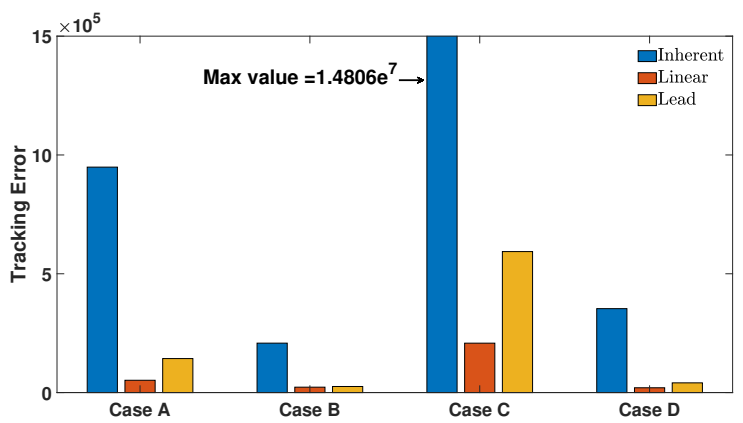

(f) Tracking error for four perturbations under consideration.

Fig. 4. Real power tracking performance of the DER subject to different disturbances.

\section{B. Case B: Induction Machine Energization}

The response of the DER subject to a switching transient because of energization of an induction machine is shown in Fig. 4c; the performance indicators are presented in Table III. The proposed linear prediction strategy is within $1 \%$ margin to lead compensator strategy for overshoot while it exhibits a $32 \%$ improvement in settling time.

\section{Case C: Grid Voltage Set Point Change}

Fig. 4d shows the response of the DER subject to two grid voltage set point changes: (i) a $10 \%$ increase in voltage at $t=0.1 \mathrm{~s}$ and back to nominal voltage at $t=2.1 \mathrm{~s}$ and (ii) a $10 \%$ decrease in voltage at $t=4.1 \mathrm{~s}$ and back to nominal voltage at $t=6.1 \mathrm{~s}$; performance indicators are presented in Table III. Both prediction strategies exhibit similar behavior 
for change in voltage set points and both offer much faster response for increase in voltage with reduced overshoot. The settling time does not experience a significant change.

\section{Case D: Impact of System Strength (SCR)}

This case study evaluates the dynamic performance of the DER subject to a change in system short circuit ratio (SCR). For 100\% inverter-penetrated microgrids, changing the virtual impedance $Z_{v}$ of individual inverters is an effective solution to improve stability [29]. Changing $Z_{v}$ in turn impacts the SCR of the system. The response of the DER subject to change in virtual impedance is shown in Fig. 4e, where $Z_{v}$ is reduced from $50 \mu \mathrm{H}$ to $5 \mu \mathrm{H}$ at $t=0.1 \mathrm{~s}$ and increased back to $50 \mu \mathrm{H}$ at $t=2.1 \mathrm{~s}$. The performance indicators presented in Table III. Again, SSPAACE improves the response of the system significantly.

\section{E. Comparison of Tracking Error}

The tracking error for the inherent controller and the controller with SSPAACE incorporating linear and lead prediction strategies is shown in Fig. 4f. SSPAACE improves the dynamic performance of DER considerably while the proposed linear prediction strategy outperforms the lead compensator prediction strategy except for the case of switching transients where the latter performs marginally better.

\section{CONCLUSIONS}

This paper discusses an add-on function to improve the performance of existing controllers when the system characteristics changes and the controlled unit is sensitive to overvoltages and overcurrents. This add-on controller, referred to as SPAACE, uses prediction of the system response to modulate its set point to achieve the desired response trajectory. This paper presents a smooth modulation strategy for SPAACE and discusses the performance of different prediction strategies, as validated based on a hardware test bed. The extensive case studies show the superior performance of a simple, linearbased prediction law in appreciably improving the dynamic response characteristics, e.g., settling time, overshoot, and tracking error, of a DER unit interfaced by power electronics.

\section{REFERENCES}

[1] D. Olivares, A. Mehrizi-Sani, A. Etemadi, C. Canizares, R. Iravani, M. Kazerani, A. Hajimiragha, O. Gomis-Bellmunt, M. Saeedifard, R. Palma-Behnke, G. Jimenez-Estevez, and N. Hatziargyriou, "Trends in microgrid control," IEEE Trans. Smart Grid, vol. 5, no. 4, pp. 19051919, Jul. 2014

[2] A. Mehrizi-Sani, "Distributed control techniques in microgrid," in Microgrid: Advanced Control Methods and Renewable Energy System Integration, M. S. Mahmoud, Ed. Elsevier, 2017.

[3] M. Yazdanian and A. Mehrizi-Sani, "Distributed control techniques in microgrids," IEEE Trans. Smart Grid, vol. 5, no. 6, pp. 2901-2909, Nov. 2014.

[4] H. Li, F. Li, Y. Xu, D. T. Rizy, and J. D. Kueck, "Adaptive voltage control with distributed energy resources: Algorithm, theoretical analysis, simulation, and field test verification," IEEE Trans. Power Syst., vol. 25, no. 3, pp. 1638-1647, Aug. 2010.

[5] W. Leithead, "Survey of gain-scheduling analysis design," Int. J. Control, vol. 73, pp. 1001-1025, 1999.
[6] P. M. Namara, R. R. Negenborn, B. D. Schutter, and G. Lightbody, "Optimal coordination of a multiple HVDC link system using centralized and distributed control," IEEE Trans. Control Syst. Technol., vol. 21, no. 2, pp. 302-314, Mar. 2013.

[7] M. Moradzadeh, R. Boel, and L. Vandevelde, "Voltage coordination in multi-area power systems via distributed model predictive control," IEEE Trans. Power Syst., vol. 28, no. 1, pp. 513-521, Feb. 2013.

[8] S. Roshany-Yamchi, M. Cychowski, R. Negenborn, B. Schutter, K. Delaney, and J. Connell, "Kalman filter-based distributed predictive control of large-scale multi-rate systems: Application to power networks," IEEE Trans. Control Syst. Technol., vol. 21, no. 1, pp. 27-39, Jan. 2013.

[9] K. J. Åström and T. Hägglund, PID Controllers: Theory, Design and Tuning, 2nd ed. Research Trinangle Park, NC: Instrum. Soc. Amer., 1995.

[10] K. J. Åström, "Auto-tuning, adaptation and expert control," in Americal Control Conf., Boston, MA, Jun. 1985, pp. 1514-1519.

[11] K. J. Åström and R. M. Murray, Feedback Systems: An Introduction for Scientists and Engineers, 2nd ed. Princeton, NJ: Princeton University Press, 2008. [Online]. Available: http://www.cds.caltech.edu/ murray/ books/AM08/pdf/am08-complete_28Sep12.pdf

[12] K. J. Åström and T. Hägglund, "Automatic tuning of PID controllers," in The Control Handbook, W. Levin, Ed. CRC Press and IEEE Press, 1996, no. 53.

[13] N. Killingsworth and M. Krstic, "PID tuning using extremum seeking," IEEE Control Syst. Mag., vol. 26, no. 1, pp. 70-79, Feb. 2006.

[14] H. Hjalmarsson, M. Gevers, S. Gunnarsson, and O. Lequin, "Iterative feedback tuning: Theory and applications," IEEE Control Syst. Mag., vol. 18, no. 4, pp. 26-41, Aug. 1998.

[15] O. Lequin, E. Bosmans, and T. Triest, "Iterative feedback tuning of PID parameters: Comparison with classical tuning rules," Contr. Eng. Pract., vol. 11, no. 9, pp. 1023-1033, Sep. 2003.

[16] O. Lequin, M. Gevers, and T. Triest, "Optimizing the settling time with iterative feedback tuning," in Proc. 15th IFAC World Cong., Beijing, China, 1993, pp. 433-437.

[17] O. J. M. Smith, "Posicast control of damped oscillatory systems," Proc. IRE, vol. 45, no. 9, pp. 1249-1255, Sep. 1957.

[18] J. Y. Hung, "Feedback control with posicast," IEEE Trans. Ind. Electron., vol. 50, no. 1, pp. 94-99, Feb. 2003.

[19] J. Vaughan, A. Smith, S. J. Kang, and W. Singhose, "Predictive graphical user interface elements to improve crane operator performance," IEEE Trans. Syst., Man, Cybern., vol. 41, no. 2, pp. 323-330, Mar. 2011.

[20] G. Cook, "An application of half-cycle posicast," IEEE Trans. Autom. Control, vol. 11, no. 3, pp. 556-559, Jul. 1966.

[21] J. Y. Hung, "Posicast control past and present," IEE Multidisciplinary Eng. Educ. M., vol. 2, no. 1, pp. 7-11, Mar. 2007.

[22] Q. Feng, R. M. Nelms, and J. Y. Hung, "Posicast-based digital control of the buck converter," IEEE Trans. Ind. Electron., vol. 53, no. 3, pp. 759-767, Jun. 2006.

[23] A. Mehrizi-Sani and R. Iravani, "Online set point adjustment for trajectory shaping in microgrid applications," IEEE Trans. Power Syst., vol. 27, no. 1, pp. 216-223, Feb. 2012.

[24] A. Mehrizi-Sani and R. Iravani, "Online set point modulation to enhance microgrid dynamic response: Theoretical foundation," IEEE Trans. Power Syst., vol. 27, no. 4, pp. 2167-2174, Nov. 2012.

[25] H. Ghaffarzadeh, C. Stone, and A. Mehrizi-Sani, "Predictive set point modulation to mitigate transients in lightly damped balanced and unbalanced systems," IEEE Trans. Power Syst., vol. 32, no. 2, pp. 1041-1049, Mar. 2017.

[26] H. Ghaffarzadeh and A. Mehrizi-Sani, "Predictive set point modulation to mitigate transients in power systems with a multiple-input-multipleoutput control system," in IEEE Innovative Smart Grid Technol. Conf. (ISGT), Minneapolis, MN, Sep. 2016.

[27] H. Ghaffarzadeh and A. Mehrizi-Sani, "A set point modulation technique with exponential prediction algorithm to improve the dynamic response of power systems," in IEEE Applied Power Electron. Conf. Expo (APEC), Tampa, FL, Mar. 2017.

[28] M. Yazdanian, A. Mehrizi-Sani, R. Seebacher, K. Krischan, and A. Muetze, "Smooth reference modulation to improve dynamic response in drive systems," IEEE Trans. Power Electron., vol. 33, no. 7, pp. 64346443, Jul. 2018.

[29] X. Lu, K. Sun, J. M. Guerrero, J. C. Vasquez, L. Huang, and J. Wang, "Stability enhancement based on virtual impedance for dc microgrids with constant power loads," IEEE Trans. Smart Grid, vol. 6, no. 6, pp. 2770-2783, Nov. 2015. 